\title{
Time Dependent Axially Symmetric Solutions of the Einstein-Maxwell-Yukawa Fields
}

\author{
A. R. Roy and C. R. Datta \\ Department of Mathematics, Indian Institute of Technology, Kharagpur, India
}

\begin{abstract}
Extending a technique developed for static fields by Janis et al. [10] to the nonstatic fields two exact solutions in the case of Einstein-Rosen metric for the interacting electromagnetic and zero mass scalar fields have been obtained.
\end{abstract}

\section{Introduction}

The need for exact solutions in the general theory of relativity is well known. In view of the highly nonlinear character of the field equations only a limited number of solutions are available in the theory. The object of this paper is to generate solutions for the interacting electromagnetic and meson fields which study is of considerable interest in the field of high energy particle physics.

The possibility of construction of new solutions out of existing solutions in some special cases has been established by the following authors. Datta Majumdar [1] has shown that for a static EinsteinMaxwell source free field the solutions could be generated from those of corresponding vacuum solutions. This investigation has further been extended by Misra and Radhakrishna [2] and later by Harrison [3] to the case of nonstatic Weyl fields. Formulating the idea of 'reciprocal solutions' Buchdahl [4], [5], [6], [7], has developed methods for generating new solutions from those of empty spacetime solutions. His work incidentally extends into Unified theories. It may be mentioned here that work relating to the generation of solutions of Unified theory has been done by Bandyopadhyay [8] and Rao and Tiwari [9].

Janis et al. [10] have developed results, for constructing solutions for the coupled fields from those of vacuum field equations in the case of a static metric. Observing that in the cylindrically symmetric metric we have considered, the results of Janis et al. [10] can be adapted to generate time dependent solutions from those of vacuum solutions, we have obtained two exact solutions. These solutions correspond to two of the solutions obtained by Rao et al. [11]. This technique is a powerful tool for obtaining new time dependent solutions and the work is being pursued separately. 


\section{Field Equations}

The general relativistic field equations for the interacting sourcefree electromagnetic and zero mass scalar (meson) field is given by

$$
G_{i j} \equiv R_{i j}-\frac{1}{2} R g_{i j}=-\kappa\left(T_{i j}^{(e)}+T_{i j}^{(s)}\right) .
$$

where $\kappa\left(=\frac{8 \pi G}{c^{4}}\right)$ is the gravitational constant.

$$
T_{i j}^{(e)} \text { given by } \frac{1}{4 \pi}\left[-F_{i s} F_{j}^{s}+\frac{1}{4} g_{i j} F_{s p} F^{s p}\right],
$$

is the energy momentum tensor for the electromagnetic field and $T_{i j}^{(s)}$ given by

$$
\frac{1}{4 \pi}\left[V_{, i} V_{, j}-\frac{1}{2} g_{i j} V_{, s} V^{, s}\right],
$$

the energy momentum tensor for the zero mass scalar field. Here $F_{i j}$ satisfies the Maxwell field equations

$$
\begin{aligned}
F_{i j} & =A_{i, j}-A_{j, i}, \\
F_{; j}^{i j} & =0,
\end{aligned}
$$

$A_{i}$ being the four potential.

Following [11], the final set of field equations for the coupled electromagnetic and zero mass (meson) scalar fields, for the axially symmetric Einstein-Rosen metric

$$
d s^{2}=e^{2 \alpha-2 \beta}\left(d t^{2}-d \varrho^{2}\right)-\varrho^{2} e^{-2 \beta} d \Phi^{2}-e^{2 \beta} d z^{2},
$$

is as follows:

$$
\begin{aligned}
\beta_{1}^{2}+\beta_{4}^{2}-\frac{\alpha_{1}}{\varrho}= & -\frac{\kappa}{8 \pi}\left[\frac{e^{2 \beta}}{\varrho^{2}}\left(\phi_{1}^{2}+\phi_{4}^{2}\right)+e^{-2 \beta}\left(\psi_{1}^{2}+\psi_{4}^{2}\right)\right] \\
& -\frac{\kappa}{8 \pi}\left(V_{1}^{2}+V_{4}^{2}\right), \\
\beta_{11}-\beta_{44}+\frac{\beta_{1}}{\varrho}= & \frac{\kappa}{8 \pi}\left[\frac{e^{2 \beta}}{\varrho^{2}}\left(\phi_{1}^{2}-\phi_{4}^{2}\right)-e^{-2 \beta}\left(\psi_{1}^{2}-\psi_{4}^{2}\right)\right], \\
\frac{\alpha_{4}}{\varrho}-2 \beta_{1} \beta_{4}= & \frac{\kappa}{4 \pi}\left(\frac{e^{2 \beta}}{\varrho^{2}} \phi_{1} \phi_{4}+e^{-2 \beta} \psi_{1} \psi_{4}\right)+\frac{\kappa}{4 \pi} V_{1} V_{4}, \\
\phi_{1} \psi_{1}-\phi_{4} \psi_{4}= & 0 \\
\phi_{11}-\phi_{44}-\frac{\phi_{1}}{\varrho}= & 2 \beta_{4} \phi_{4}-2 \beta_{1} \phi_{1}, \\
\psi_{11}-\psi_{44}+\frac{\psi_{1}}{\varrho}= & 2 \beta_{1} \psi_{1}-2 \beta_{4} \psi_{4}
\end{aligned}
$$


and

$$
V_{11}-V_{44}+\frac{V_{1}}{\varrho}=0
$$

where $\phi$ and $\psi$ denote the components $A_{2}$ and $A_{3}$ of the electromagnetic 4-potential and $V$ is the scalar potential satisfying the wave equation

$$
g^{i j} V_{; i j}=0 \text {. }
$$

Here $\alpha, \beta, \phi, \psi$ and $V$ are functions of $\varrho$ and $t$ only and the suffixes 1 and 4 after these unknown functions denote partial differentiation with respect to $\varrho$ and $t$ respectively.

\section{Technique for Developing the Solutions}

The results of Janis et al. [10] modified for a metric of signature $(--++)$ read as follows:

If a vacuum solution of the Einstein's field equations is given by the metric of the line element

$$
d s^{2}=e^{2 v}\left(d x^{4}\right)^{2}-e^{-2 v} h_{i j} d x^{i} d x^{j},
$$

then a static solution of the coupled Einstein scalar field equations is given by the metric of the line element

$$
d s^{2}=e^{2 u}\left(d x^{4}\right)^{2}-e^{-2 u} h_{i j} d x^{i} d x^{j}
$$

and $V$ where

$$
V=A u \quad \text { and } \quad u=v\left(1+\frac{\kappa A^{2}}{8 \pi}\right)^{-\frac{1}{2}},
$$

$A$ being a constant. If in addition to the scalar field a sourcefree electromagnetic field is also taken then the solutions of the coupled Einstein Maxwell Yukawa field equations

$$
\begin{gathered}
G_{i j} \equiv R_{i j}-\frac{1}{2} R g_{i j}=-\frac{\kappa}{4 \pi}\left(V_{, i} V_{, j}-\frac{1}{2} g_{i j} V_{, s} V^{s}-F_{i s} F_{j}^{s}+\frac{1}{4} g_{i j} F_{s p} F^{s p}\right), \\
\square V=0, \quad F_{; j}^{i j}=0, \quad F_{[i j, K]}=0,
\end{gathered}
$$

is given by

$$
V, F_{i j}=\left(\frac{8 \pi}{\kappa}\right)^{\frac{1}{2}} e^{2 w}\left(\delta_{i}^{4} u_{, j}-\delta_{j}^{4} u_{, i}\right)
$$

and the metric of the line element

where

$$
d s^{2}=e^{2 w}\left(d x^{4}\right)^{2}-e^{-2 w} h_{i j} d x^{i} d x^{j},
$$

$$
w=-\log \cos h u \text {. }
$$


As this theorem is true in general for the static fields only, we shall in the following section first convert the nonstatic metric (2.6) to a static metric by the transformation $(z, t) \rightarrow\left(i t^{\prime}, i z^{\prime}\right)$ and then apply the technique to get a solution in the static form. Finally applying the reverse transformation $\left(z^{\prime}, t^{\prime}\right) \rightarrow(i t, i z)$ we get the solution of the coupled field in the nonstatic form. The above mentioned conversion of nonstatic fields to the corresponding static fields and vice versa is possible only in the case of axially symmetric fields. It may also be remarked that the results of [10] presupposes the form for $d s^{2}$ given by (3.1) which is possible in general only in the case of static fields.

\section{Applications of the Technique}

In this section, we shall generate the solutions of the field equations (2.7)-(2.13) for the coupled fields from the known solutions of $R_{i j}=0$, by applying the technique mentioned in the preceeding section.

\section{(i) A Time Independent Solution:}

The field equations for the empty space characterized by $R_{i j}=0$, for the metric (2.6) become

$$
\begin{aligned}
\beta_{1}^{2}+\beta_{4}^{2}-\frac{\alpha_{1}}{\varrho} & =0, \\
\beta_{11}-\beta_{44}+\frac{\beta_{1}}{\varrho} & =0, \\
\frac{\alpha_{4}}{\varrho}-2 \beta_{1} \beta_{4} & =0 .
\end{aligned}
$$

The solution of (4.1)-(4.3) obtained by Levi-Civita [12] is given by the metric

$$
d s^{2}=\left(\frac{\varrho}{\varrho_{0}}\right)^{\frac{q^{2}+2 q}{2}}\left(d t^{2}-d \varrho^{2}\right)-\left(\frac{\varrho}{\varrho_{0}}\right)^{q} \varrho^{2} d \Phi^{2}-\left(\frac{\varrho}{\varrho_{0}}\right)^{-q} d z^{2},
$$

where $\varrho_{0}$ is a constant. This solution corresponds to a line mass placed along the $z$-axis.

Applying the transformation

$$
\varrho \rightarrow \varrho^{\prime}, \quad \Phi \rightarrow \Phi^{\prime}, \quad z \rightarrow i t^{\prime}, \quad t \rightarrow i z^{\prime},
$$

the metric (4.4) becomes

$$
d s^{2}=\left(\frac{\varrho^{\prime}}{\varrho_{0}}\right)^{-q} d t^{\prime 2}-\left(\frac{\varrho^{\prime}}{\varrho_{0}}\right)^{q}\left[\left(d \varrho^{\prime 2}+d z^{\prime 2}\right)\left(\frac{\varrho}{\varrho_{0}}\right)^{\frac{q}{2}}+\varrho^{\prime 2} d \Phi^{\prime 2}\right] .
$$


Identifying (4.6) with the metric (3.1), we have

and

$$
v=\frac{q}{2} \log \left(\frac{\varrho_{0}}{\varrho^{\prime}}\right)
$$

$$
h_{i j} d x^{i} d x^{j}=\left(\frac{\varrho^{\prime}}{\varrho_{0}}\right)^{\frac{q^{2}}{2}}\left(d \varrho^{\prime 2}+d z^{\prime 2}\right)+\varrho^{\prime 2} d \Phi^{\prime 2} \text {. }
$$

From (3.3), we get the scalar potential $V$ as

i. e.,

$$
V=A \frac{q}{2}\left(1+\frac{\kappa A^{2}}{8 \pi}\right)^{-\frac{1}{2}} \log \left(\frac{\varrho_{0}}{\varrho^{\prime}}\right),
$$

where

$$
V=A M \log \left(\frac{\varrho_{0}}{\varrho^{\prime}}\right) \text {, }
$$

$$
M=\frac{q}{2}\left(1+\frac{\kappa A^{2}}{8 \pi}\right)^{-\frac{1}{2}} \text {. }
$$

Again from (3.5), we have

$$
\begin{aligned}
w & =-\log \cosh u \\
& =\log \operatorname{sech}\left(N-M \log \varrho^{\prime}\right),
\end{aligned}
$$

where $N=M \log \varrho_{0}$, is a constant.

This gives

$$
e^{2 w}=\operatorname{sech}^{2}\left(N-M \log \varrho^{\prime}\right) .
$$

We shall now find the electromagnetic field. It is clear from the structure of (3.4) that the only nonzero component of the electromagnetic 4-potential is $F_{14}$.

Thus from (3.4), we have

$$
\begin{aligned}
F_{14} & =-\left(\frac{8 \pi}{\kappa}\right)^{\frac{1}{2}} e^{2 w} \frac{\partial}{\partial \varrho^{\prime}}\left\{M \log \left(\frac{\varrho_{0}}{\varrho^{\prime}}\right)\right\}, \\
& =-\left(\frac{8 \pi}{\kappa}\right)^{\frac{1}{2}} \frac{M}{\varrho^{\prime}} \operatorname{sech}^{2}\left(N-M \log \varrho^{\prime}\right) .
\end{aligned}
$$

Noting $F_{14}=-\psi_{1}$, we have

$$
\psi_{1}=\left(\frac{8 \pi}{\kappa}\right)^{\frac{1}{2}} \frac{M}{\varrho^{\prime}} \operatorname{sech}^{2}\left(N-M \log \varrho^{\prime}\right),
$$

which on integration gives

$$
\psi=p+\left(\frac{8 \pi}{\kappa}\right)^{\frac{1}{2}} \tanh \left(N-M \log \varrho^{\prime}\right) .
$$


Now applying the reverse transformation

$$
\varrho^{\prime} \rightarrow \varrho, \quad \Phi^{\prime} \rightarrow \Phi, \quad t^{\prime} \rightarrow i z, \quad z^{\prime} \rightarrow i t,
$$

in (4.8), (4.10) and (4.11), we get the final solution of the coupled field in the form

and $g_{i j}$ given by

$$
V=A M \log \left(\frac{\varrho_{0}}{\varrho}\right), \quad \psi=p+\left(\frac{8 \pi}{\kappa}\right)^{\frac{1}{2}} \tanh (N-M \log \varrho)
$$

$$
\begin{aligned}
d s^{2}= & \cosh ^{2}(N-M \log \varrho)\left(\frac{\varrho}{\varrho_{0}}\right)^{\frac{q^{2}}{2}}\left(d t^{2}-d \varrho^{2}\right) \\
& -\varrho^{2} \cosh ^{2}(N-M \log \varrho) d \Phi^{2}-\operatorname{sech}^{2}(N-M \log \varrho) d z^{2} .
\end{aligned}
$$

Making the identification of (4.13) with (2.6), we get

$$
\begin{aligned}
& \alpha=\frac{q^{2}}{4} \log \left(\frac{\varrho}{\varrho_{0}}\right), \\
& \beta=\frac{1}{2} \log \left\{\operatorname{sech}^{2}(N-M \log \varrho)\right\} .
\end{aligned}
$$

Adjusting the arbitrary constants involved, this solution can easily be identified with the solution (41) of [11].

(ii) A Nonstatic Solution:

We now consider a time dependent solution of the empty space field equations (4.1)-(4.3) given by (Misra and Radhakrishna [2])

$$
d s^{2}=e^{\frac{q^{2} \varrho^{2}}{4}+a+q t-n}\left(d t^{2}-d \varrho^{2}\right)-\varrho^{2} e^{q t-n} d \Phi^{2}-e^{-q t+n} d z^{2},
$$

where $\varrho, n$ and $a$ are arbitrary constants.

As in the previous solution, applying the transformation (4.5) and then identifying the transformed metric with (3.1), we get

and

$$
v=-\frac{1}{2}\left(i q z^{\prime}-n\right)
$$

$$
h_{i j} d x^{i} d x^{j}=e^{\frac{q^{2} \varrho^{\prime 2}}{4}+a}\left(d \varrho^{\prime 2}+d z^{\prime 2}\right)+\varrho^{\prime 2} d \Phi^{\prime 2} .
$$

Thus in this case the scalar potential is given by

i. e.,

$$
V=A\left(1+\frac{\kappa A^{2}}{8 \pi}\right)^{-\frac{1}{2}}\left\{-\frac{1}{2}\left(i q z^{\prime}-n\right)\right\},
$$

$$
V=-A M\left(i q z^{\prime}-n\right) \text {, }
$$


where now

$$
M=\frac{1}{2}\left(1+\frac{\kappa A^{2}}{8 \pi}\right)^{-\frac{1}{2}} .
$$

Also the metric characterising the solution of the coupled field equations is

$$
\begin{aligned}
d s^{2}= & \operatorname{sech}^{2}\left\{-M\left(i q z^{\prime}-n\right)\right\} d t^{\prime 2} \\
& -\cosh ^{2}\left\{-M\left(i q z^{\prime}-n\right)\right\}\left[e^{\frac{q^{2}{Q^{\prime}}^{2}}{4}+a}\left(d \varrho^{\prime 2}+d z^{\prime 2}\right)+\varrho^{\prime 2} d \Phi^{\prime 2}\right] .
\end{aligned}
$$

Again from (3.4), the only component of the electromagnetic field tensor which survives in this case is given by

$$
F_{34}=-\left(\frac{8 \pi}{\kappa}\right)^{\frac{1}{2}} e^{2 w} \frac{\partial}{\partial z^{\prime}}\left\{-M\left(i q z^{\prime}-n\right)\right\},
$$

which on simplification gives

$$
F_{34}=\left(\frac{8 \pi}{\kappa}\right)^{\frac{1}{2}} i q M \operatorname{sech}^{2}\left\{-M\left(i q z^{\prime}-n\right)\right\} .
$$

Assuming $F_{34}=\psi_{3}$, and integrating w.r.t. $z^{\prime}$ we get

$$
\psi=p-\left(\frac{8 \pi}{\kappa}\right)^{\frac{1}{2}} \tanh \left\{-M\left(i q z^{\prime}-n\right)\right\} .
$$

Now applying the reverse transformation (4.12) in (4.16), (4.17) and (4.18), we get

and the $g_{i j}$ given by

$$
\begin{aligned}
& V=A M(q t+n) \\
& \psi=p-\left(\frac{8 \pi}{\kappa}\right)^{\frac{1}{2}} \tanh \{M(q t+n)\}
\end{aligned}
$$

$$
\begin{aligned}
d s^{2}= & e^{\frac{q^{2} \varrho^{2}}{4}+a} \cosh ^{2}\{M(q t+n)\}\left(d t^{2}-d \varrho^{2}\right) \\
& -\cosh ^{2}\{M(q t+n)\} \varrho^{2} d \Phi^{2}-\operatorname{sech}^{2}\{M(q t+n)\} d z^{2} .
\end{aligned}
$$

Identifying (4.21) with (2.6), we have

$$
\begin{aligned}
& \alpha=e^{a} \frac{q^{2} \varrho^{2}}{8}, \\
& \beta=\frac{1}{2} \log \left\{\operatorname{sech}^{2} M(q t+n)\right\} .
\end{aligned}
$$

This solution also after certain adjustments of the arbitrary constants corresponds to the solution (50) of [11].

Acknowledgements: The authors are grateful to Dr. J. R. Rao for suggesting the problem and his help in the preparation of this paper. They also thank Prof. G. Bandyopadhyay and Dr. R. N. Tiwari for some helpful remarks. 


\section{References}

1. Datta Majumdar, S.: Phys. Rev. 72, 390 (1947).

2. Misra, M., Radhakrishna, L.: Proc. Nat. Inst. Sci. India, Part A, 28, 632 (1962).

3. Harrison, B. K.: Phys. Rev. 138 B, 488 (1965).

4. Buchdahl, H.: Quart. J. Maths. 5, 116 (1954).

5. Buchdahl, H.: Aust. J. Phys. 9, 13 (1956).

6. Buchdahl, H.: Nuovo Cimento Ser. X, 5, 1083 (1957).

7. Buchdahl, H.: Phys. Rev. 115, 1325 (1959).

8. Bandyopadhyay, G.: J. Math. Mech. 12, 655 (1963).

9. Rao, J.R., Tiwari, R. N.: Communicated for publication.

10. Janis, A.I., Robinson, D. C., Winicour, J.: Phys. Rev. 186, 1729 (1969).

11. Rao,J.R., Roy, A.R., Tiwari, R. N.: Ann. Phys. 69, 473 (1972).

12. Levi-Civita, T.: R. C. Acad. Lincei, 28i, 101 (1919).

A. R. Roy

Dept. of Mathematics

Indian I'nstitute of Technology

Kharagpur-2/India 